Artículo

\title{
Impacto de diferentes fertilizantes en la solución del suelo y el crecimiento de tomate
}

\author{
Jorge Enrique Canales-Almendares ${ }^{1}$ \\ Fernando Borrego-Escalante ${ }^{2}$ \\ Willian Alfredo Narvaez-Ortíz ${ }^{1}$ \\ Susana González-Morales ${ }^{3}$ \\ Adalberto Benavides-Mendoza ${ }^{4 \S}$
}

${ }^{1}$ Doctorado en Agricultura Protegida-Universidad Autónoma Agraria Antonio Narro. Saltillo, Coahuila, México. CP. 25315. (jorgecanales15@yahoo.es; williamnarvaezo@gmail.com). ${ }^{2}$ Departamento de Fitomejoramiento-Universidad Autónoma Agraria Antonio Narro. Saltillo, Coahuila, México. CP. 25315. (fborregoe9@gmail.com). ${ }^{3}$ CONACYT-UAAAN-Departamento de Horticultura. Saltillo, Coahuila, México. CP. 25315. (qfb_sgm@hotmail.com). ${ }^{4}$ Departamento de Horticultura-Universidad Autónoma Agraria Antonio Narro. Calzada Antonio Narro núm. 1923. Saltillo, Coahuila, México. CP. 25315. (abenmen@gmail.com).

${ }^{\S}$ Autor para correspondencia: abenmen@gmail.com.

\section{Resumen}

La solución del suelo es un sistema complejo y abierto que tiene entradas y salidas de energía, este estudio se llevó a cabo para determinar el impacto de diferentes tipos de fertilizantes en la composición de la solución del suelo y la acumulación de biomasa en plantas de tomate. Se utilizaron plantas de tomate cultivadas en invernadero en macetas con suelo calcáreo y suelo forestal, para aplicar tres tipos de fertilizantes: solución de Steiner, fertilizantes sólidos y té de vermicomposta. Las concentraciones de $\mathrm{NO}_{3}{ }^{-}, \mathrm{K}^{+}, \mathrm{Ca}^{2+}, \mathrm{Mg}^{2+}, \mathrm{Na}^{+}$y $\mathrm{SO}_{4}{ }^{2-}$ en la solución del suelo aumentaron con el tiempo, registrándose mayores valores de concentración de iones en el fertilizante sólido $\left(625,183,374,70,49\right.$ y $161 \mathrm{mg} \mathrm{L}^{-1}$, respectivamente) y en la solución de Steiner (500, 177, 363, 65, 69 y $\left.235 \mathrm{mg} \mathrm{L}^{-1}\right)$. Las concentraciones más altas de iones en la solución del suelo coincidieron con la mayor acumulación de biomasa, 3938 y 4546 g planta $^{-1}$ en el fertilizante sólido y en la solución Steiner respectivamente. En contraste, en la solución de suelo de té de vermicomposta, a excepción del $\mathrm{Na}^{+}$, hubo concentraciones de iones más bajas $(94,63,118,28$, 75 y $98 \mathrm{mg} \mathrm{L}^{-1}$ ) y una menor acumulación de biomasa de $1355 \mathrm{~g} \mathrm{planta}^{-1}$. Los tratamientos de solución Steiner y fertilizantes sólidos presentaron los valores medios más altos de rendimiento, con 3637 y 2712 g planta $^{-1}$ respectivamente. Este resultado indica que la forma de aplicación y la fuente del fertilizante influye en la solución del suelo.

Palabras clave: Solanum lycopersicum L., aniones, calcisoles, cationes, nutrición mineral.

Recibido: enero de 2021

Aceptado: abril de 2021 


\section{Introducción}

Las plantas toman de la solución del suelo (SS) los nutrientes necesarios para su desarrollo y crecimiento, los elementos que se encuentra en la SS y que están en contacto con las raíces de la planta se ven influenciados por muchos procesos como, la respiración microbiana, los exudados de la raíz, la absorción, fijación por las partículas del suelo, el drenaje, la cantidad de oxígeno, temperatura, humedad del suelo, los procesos redox etc. Son pocos los estudios que se han realizado en donde se aborde la influencia de los procesos antes mencionados en la dinámica y composición de la SS a través del tiempo, sobre todo los suelos con fines de cultivo (Huang et al., 2011).

Los procesos de intercambio son regulados a menudo por la concentración de $\mathrm{NO}_{3}{ }^{-}$, usualmente el anión dominante en la SS (Plett et al., 2020). Sin embargo, otros elementos incluidos en los fertilizantes también modifican la composición de la SS (Narváez-Ortiz et al., 2015). Por lo tanto, la forma química del fertilizante afectará directa e indirectamente la composición química de la SS y consecuentemente, la absorción de nutrientes y el crecimiento de las plantas.

El estudio de la SS permite un acercamiento al estado real de la disponibilidad de los nutrimentos liberados por los fertilizantes y los contenidos en el suelo (Hernández-Díaz et al., 2014). La SS es la interface entre la fase sólida del suelo y las células de las raíces que absorben los nutrientes. Se considera importante entender las condiciones dinámicas prevalecientes en la SS, ya que los cambios en la composición pueden ocurrir rápidamente, especialmente durante la absorción de nutrientes por las plantas (Narváez-Ortiz et al., 2015). El estudio de la composición química de la SS puede llevarse a cabo por diferentes técnicas, una de ellas es la colocación de sondas de succión que permiten la extracción y caracterización en el laboratorio o incluso in situ de la muestra de SS.

El estudio del comportamiento dinámico de la SS permite establecer un índice de disponibilidad de nutrientes minerales, así como su impacto en los cultivos (Souza et al., 2013). La solución del suelo es la fase dinámica que conecta el uso de los fertilizantes con la lixiviación y la adsorción en la fase sólida del suelo y con la absorción y el crecimiento de las plantas (Llanderal et al., 2019). Hasta donde sabemos, el impacto en distintos suelos de diferentes fertilizantes sobre la composición de la solución del suelo no ha sido estudiado para el tomate bajo invernadero. El objetivo del trabajo fue evaluar el comportamiento dinámico de la solución del suelo en dos tipos de suelo con tres tipos de fertilización mineral u orgánica, y su impacto sobre la acumulación de biomasa y composición química de plantas de tomate.

\section{Materiales y métodos}

La presente investigación se llevó a cabo en la Universidad Autónoma Agraria Antonio Narro, en Saltillo, México. Se utilizaron como material experimental plantas de tomate (Solanum lycopersicum) hibrido de la variedad Toro, con hábito de crecimiento determinado. Después de la germinación y el cuidado de las plántulas, estas se establecieron bajo invernadero durante el periodo octubre-abril en macetas de polietileno de 12 L con dos tipos de suelo: un suelo calcáreo no agrícola colectado del perfil 0-0.3 m en un área sin presencia de vegetación y un suelo negro de bosque colectado del perfil 0-0.3 m, eliminando el mantillo superficial, en un área con reforestación de 20 años con Pinus halepensis. Los suelos fueron recolectados en sitios marcados por las coordenadas $25^{\circ} 21^{\prime} 14.87^{\prime \prime}$ latitud norte y $101^{\circ} 2^{\prime} 23.25^{\prime}$ ' longitud oeste para el suelo calcáreo y $25^{\circ} 21^{\prime} 6.81^{\prime \prime}$ latitud norte y $101^{\circ} 1^{\prime} 27.69^{\prime \prime}$ longitud oeste para el suelo negro de bosque. 
Antes de su uso en las macetas los suelos fueron cribados para eliminar piedras y otros materiales. Asimismo, los suelos fueron caracterizados desde el punto de vista fisicoquímico de acuerdo con la NOM-021-RECNAT-2000 (SEMARNAT 2002) (Cuadro 1). También se analizó el agua de riego con el fin de tomar en cuenta la composición de los diferentes iones para el cálculo de la aplicación de los fertilizantes (Cuadro 2). Los tratamientos iniciaron a los siete días después del trasplante (ddt) y consistieron en tres tipos de aporte nutrimental al suelo durante 22 semanas de crecimiento de las plantas. Los tres tratamientos se describen a continuación.

Cuadro 1. Propiedades fisicoquímicas de los suelos.

\begin{tabular}{|c|c|c|c|c|c|c|}
\hline Suelo & $\begin{array}{l}\mathrm{pH}^{\mathrm{a}} \\
\mathrm{H}_{2} \mathrm{O}\end{array}$ & $\mathrm{CE}\left(\mathrm{dS} \mathrm{m} \mathrm{m}^{-1}\right)$ & $\begin{array}{c}\text { Densidad } \\
\text { aparente }\left(\mathrm{g} \mathrm{cm}^{-3}\right)\end{array}$ & $\begin{array}{l}\text { Carbonatos } \\
\text { totales }(\%)\end{array}$ & $\begin{array}{l}\mathrm{N}-\mathrm{NO}_{3}^{-} \\
\left(\mathrm{mg} \mathrm{L}^{-1}\right)\end{array}$ & $\begin{array}{l}\text { P-Olsen } \\
\left(\mathrm{mg} \mathrm{L}^{-1}\right)\end{array}$ \\
\hline Calcáreo & 8.08 & 0.9 & 1.04 & 39.8 & 23.1 & 19 \\
\hline \multirow[t]{3}{*}{ Bosque } & 7.59 & 0.58 & 0.95 & 29.7 & 2.72 & 15 \\
\hline & \multicolumn{4}{|c|}{ Cationes intercambiables $\left(\mathrm{mg} \mathrm{L}^{-1}\right)$} & Textura & $\begin{array}{l}\text { Materia } \\
\text { orgánica }\end{array}$ \\
\hline & $\mathrm{Ca}^{2+}$ & $\mathrm{Mg}^{2+}$ & $\mathrm{Na}^{+}$ & $\mathrm{K}^{+}$ & Clase & $\%$ \\
\hline Calcáreo & 6536 & 714 & 107 & 296 & Franco & 0.97 \\
\hline Bosque & 6364 & 341 & 101 & 599 & Franco & 4.9 \\
\hline
\end{tabular}

${ }^{\mathrm{a}}=\mathrm{pH}(1: 2$ agua $)$.

Tratamiento 1. Testigo. Aporte continuo de una solución nutritiva Steiner (Steiner, 1961) mediante el riego. De 0-40 ddt la solución nutritiva se aplicó en concentración de $25 \%,\left(500 \mu \mathrm{S} \mathrm{cm}^{-1}\right)$ durante la etapa vegetativa, de $41-60$ ddt se elevó a 50\% (1 $\left.000 \mu \mathrm{S} \mathrm{cm}^{-1}\right)$ durante la etapa de floración, de 61-100 ddt se incrementó al 100\% (2 $\left.000 \mu \mathrm{S} \mathrm{cm}^{-1}\right)$ durante el amarre de fruto 60 ddt y 120\% (2 $400 \mu \mathrm{S} \mathrm{cm}^{-1}$ ) al iniciar la cosecha.

Tratamiento 2. Aporte semanal de nutrientes mediante fertilizantes sólidos. La cantidad aplicada fue equivalente, en términos de elementos minerales sólidos, a lo aplicado en el tratamiento 1 por medio de la solución Steiner.

Tratamiento 3. Aporte orgánico mediante el riego, con un té de vermicomposta de origen vacuno con una CE de $2000\left(\mu \mathrm{S} \mathrm{cm}^{-1}\right)$. La CE fue ajustada por dilución hasta igualar a la CE de la solución Steiner aplicada en las diferentes etapas enumeradas para el tratamiento 1. La composición del té de vermicomposta se muestra en la Cuadro 2.

Cuadro 2. Características y concentración de elementos del agua de riego y el té de vermicomposta utilizados en el experimento.

\begin{tabular}{ccccc}
\hline \multicolumn{2}{c}{ Agua de riego } & & \multicolumn{2}{c}{ Té de vermicomposta } \\
\cline { 1 - 2 } \cline { 5 - 5 } Variable & Valor & & Variable & Valor \\
\hline $\mathrm{pH}$ & 7.06 & & $\mathrm{~N}_{-} \mathrm{NO}_{3}^{-}$ & $50 \mathrm{mg} \mathrm{L}^{-1}$ \\
$\mathrm{CE}$ & $4.96 \mathrm{mg} \mathrm{L}^{-1}$ & & $\mathrm{P}$ & $9.3 \mathrm{mg} \mathrm{L}^{-1}$ \\
$\mathrm{~N}^{-N O} \mathrm{NO}_{3}$ & $6.24 \mathrm{mg} \mathrm{L}^{-1}$ & & $\mathrm{~K}^{+}$ & $440.2 \mathrm{mg} \mathrm{L}^{-1}$ \\
$\mathrm{~K}^{+}$ & & & $\mathrm{Ca}^{2+}$ & $96.5 \mathrm{mg} \mathrm{L}^{-1}$ \\
\hline
\end{tabular}




\begin{tabular}{|c|c|c|c|}
\hline \multicolumn{2}{|c|}{ Agua de riego } & \multicolumn{2}{|c|}{ Té de vermicomposta } \\
\hline Variable & Valor & Variable & Valor \\
\hline & & $\mathrm{Mg}^{2+}$ & $42.8 \mathrm{mg} \mathrm{L}^{-1}$ \\
\hline $\mathrm{Ca}^{2+}$ & $95.8 \mathrm{mg} \mathrm{L}^{-1}$ & $\mathrm{Na}^{+}$ & $186 \mathrm{mg} \mathrm{L}^{-1}$ \\
\hline $\mathrm{Mg}^{2+}$ & $24.1 \mathrm{mg} \mathrm{L}^{-1}$ & $\mathrm{SO}_{4}^{-2}$ & $154.5 \mathrm{mg} \mathrm{L}^{-1}$ \\
\hline $\mathrm{Na}^{+}$ & $20.5 \mathrm{mg} \mathrm{L}^{-1}$ & $\mathrm{Fe}^{2+}$ & $0.3 \mathrm{mg} \mathrm{L}^{-1}$ \\
\hline $\mathrm{SO}_{4}^{-2}$ & $81.7 \mathrm{mg} \mathrm{L}^{-1}$ & $\mathrm{Zn}^{2+}$ & $0.05 \mathrm{mg} \mathrm{L}^{-1}$ \\
\hline $\mathrm{HCO}_{3}^{-}$ & $256 \mathrm{mg} \mathrm{L}^{-1}$ & B & $1.2 \mathrm{mg} \mathrm{L}^{-1}$ \\
\hline $\mathrm{CO}_{3}{ }^{2-}$ & $34.2 \mathrm{mg} \mathrm{L}^{-1}$ & $\mathrm{Cu}^{2+}$ & $<0.005 \mathrm{mg} \mathrm{L}^{-1}$ \\
\hline & & $\mathrm{Mn}^{2+}$ & $<0.025 \mathrm{mg} \mathrm{L}^{-1}$ \\
\hline
\end{tabular}

Para determinar los cambios temporales en la composición de la SS se llevaron a cabo 21 muestreos de la solución del suelo, uno cada semana, usando tres repeticiones para cada tratamiento. Para la caracterización de la SS se ubicaron tres sondas de succión en cada tratamiento. Se eligieron en cada muestreo tres plantas al azar para colocar las sondas en el interior de la maceta a $15 \mathrm{~cm}$ de profundidad y $10 \mathrm{~cm}$ de la planta. Para tomar la muestra de SS se siguió el siguiente procedimiento: se aplicaron por maceta $2 \mathrm{~L}$, transcurridos 15 minutos después del riego aplicado durante la mañana se aplicó una presión de succión de 75 centibares a la sonda de succión. Al día siguiente durante la mañana se extrajo la muestra de SS de la sonda con una jeringa. El volumen de la muestra obtenida en cada sonda osciló entre 20 y $30 \mathrm{ml}$.

Las muestras obtenidas fueron sometidas primeramente a un análisis in situ para determinar: $\mathrm{pH}$ con un potenciómetro de la marca Horiba modelo B-713, la conductividad eléctrica (EC) con un equipo de la marca Horiba modelo Spectrum Cardy Twin, el potencial de óxido reducción (ORP) se midió con un electrodo de la marca OMEGA modelo PHH-7011 y la concentración de nitratos $\left(\mathrm{NO}_{3}{ }^{-}\right)$fue determinada mediante un equipo de ion-selectivo de la marca Horiba modelo B-743. Posteriormente las muestras de SS fueron analizadas en el laboratorio para determinar el contenido de nitrógeno total $(\mathrm{N})$ mediante la técnica de micro Kjeldahl (AOAC, 1980), la de $\mathrm{P}$ por espectrofotometría visible (AOAC 1980$)$, y la concentración de potasio $\left(\mathrm{K}^{+}\right)$, calcio $\left(\mathrm{Ca}^{2+}\right)$, magnesio $\left(\mathrm{Mg}^{2+}\right)$, sulfato $\left(\mathrm{SO}_{4}^{-2}\right)$, sodio $\left(\mathrm{Na}^{+}\right)$y boro (B) un equipo ICP-OES de la marca Perkin Elmer modelo optima 8300.

Las plantas utilizadas en el experimento fueron colocadas en densidad de 3 plantas $\mathrm{m}^{-2}$, fueron mantenidas en el invernadero durante 22 semanas después de su trasplante y recibieron las labores culturales como tutoreo, poda, monitoreo y control de plagas y enfermedades. Sobre las plantas se hicieron tres muestreos colectando tres plantas al azar en cada tratamiento a los 40,60 y $90 \mathrm{ddt}$. Las plantas fueron separadas en tallos, hojas y raíces para determinar su peso fresco usando una balanza digital. Las muestras frescas fueron colocadas en bolsas de papel etiquetadas y se secaron en una estufa de deshidratación a $70{ }^{\circ} \mathrm{C}$ durante $72 \mathrm{~h}$ hasta peso constante para luego determinar el peso seco (PS) usando una balanza digital.

El arreglo estadístico utilizado fue completamente al azar con 30 repeticiones por tratamiento. La unidad experimental fue una planta en una maceta, con un total de 180 plantas en el experimento. Los resultados de la composición mineral de la solución del suelo se presentaron en graficas de dispersión contra el tiempo. Los datos de la biomasa de la planta se analizaron con el software R, para cada una de las variables de la biomasa se realizó un análisis de varianza y la prueba de medias de Fisher con un valor de significancia de $p \leq 0.05$. 


\section{Resultados y discusión}

Producción de fruto por planta. De acuerdo con el análisis de varianza, se presentaron diferencias significativas $(p \leq 0.05)$ en la cantidad de fruto por planta entre las distintas formas de aplicar los fertilizantes (Figura 1). Las diferencias entre la concentración de iones en la SS y la acumulación de biomasa a través del tiempo (Cuadro 3-4) impactaron en la producción del fruto (Figura 1).

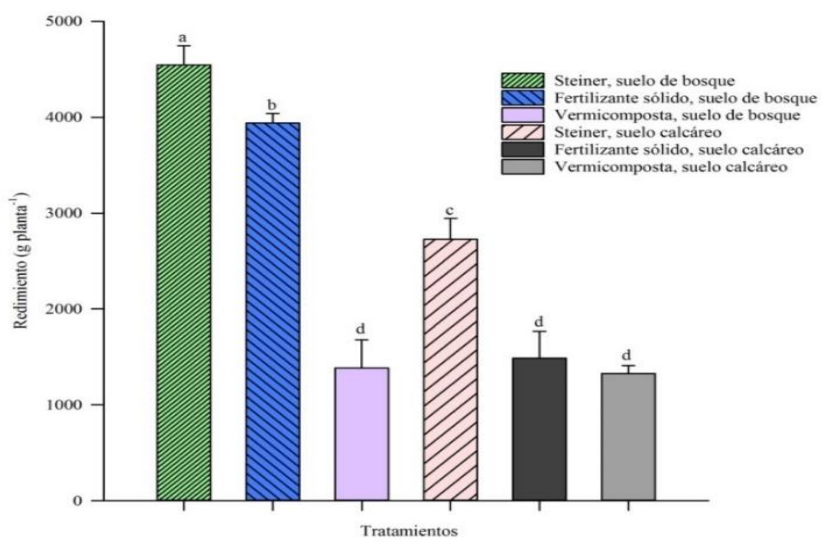

Figura 1. Valores medios de rendimiento $\left(\mathrm{g} \mathrm{planta}^{-1}\right)$ cuantificados en el ciclo del cultivo para dos clases de suelo con diferentes tipos de aplicación de fertilizantes en plantas de tomate. Significancia $p \leq 0.05$ DLS Fisher.

Cuadro 3. Concentración promedio de elementos en la solución para dos tipos de suelos.

\begin{tabular}{cccccccc}
\hline Suelo & T. Fertilizante & $\begin{array}{c}\mathrm{Ca}^{2+} \\
\left(\mathrm{mg} \mathrm{L}^{-1}\right)\end{array}$ & $\begin{array}{c}\mathrm{Mg}^{2+} \\
\left(\mathrm{mg} \mathrm{L}^{-1}\right)\end{array}$ & $\begin{array}{c}\mathrm{Na}^{+} \\
\left(\mathrm{mg} \mathrm{L}^{-1}\right.\end{array}$ & $\begin{array}{c}\mathrm{SO}_{4}^{-2} \\
\left(\mathrm{mg} \mathrm{L}^{-1}\right)\end{array}$ & $\begin{array}{c}\mathrm{K}^{+} \\
\left(\mathrm{mg} \mathrm{L}^{-1}\right)\end{array}$ & $\begin{array}{c}\mathrm{NO}_{3}^{-} \\
\left(\mathrm{mg} \mathrm{L}^{-1}\right)\end{array}$ \\
\hline Bosque & Steiner & 363.69 & 65.08 & 69.75 & 235.9 & 177.67 & 500.9 \\
& Sólido & 374.03 & 70.62 & 49.11 & 161.51 & 183 & 625.55 \\
Calcáreo & Vermicomposta & 118.98 & 28.39 & 73.84 & 98.63 & 63.37 & 94.51 \\
& Steiner & 263.85 & 120.01 & 75.31 & 256.22 & 173.54 & 505.4 \\
& Sólido & 238.99 & 97.65 & 49.14 & 143.22 & 207.61 & 698.3 \\
& Composta & 45.47 & 48.57 & 64.67 & 82.811 & 65.95 & 42 \\
\hline
\end{tabular}

Cuadro 4. Acumulación de biomasa fresca y seca en tres muestreos de tejido vegetal del cultivo de tomate con tres diferentes regímenes de fertilidad y su significancia estadística.

\begin{tabular}{cccccccc}
\hline \multirow{2}{*}{ Suelo } & T. Fertilizante & \multicolumn{6}{c}{ Biomasa fresca $(\mathrm{g})$} \\
\cline { 3 - 8 } & & $(40 \mathrm{ddt})$ & $(60 \mathrm{ddt})$ & $(90 \mathrm{ddt})$ & $(40 \mathrm{ddt})$ & $(60 \mathrm{ddt})$ & $(90 \mathrm{ddt})$ \\
\hline \multirow{2}{*}{ Bosque } & Steiner & $297.5 \mathrm{a}$ & $978.04 \mathrm{a}$ & $1181.4 \mathrm{a}$ & $35.5 \mathrm{a}$ & $120.2 \mathrm{a}$ & $185.9 \mathrm{a}$ \\
& Sólido & $147.8 \mathrm{c}$ & $490.03 \mathrm{bc}$ & $631.9 \mathrm{bc}$ & $21.4 \mathrm{~b}$ & $65.4 \mathrm{bc}$ & $87.5 \mathrm{bc}$ \\
& Vermicomposta & $82 \mathrm{de}$ & $159.63 \mathrm{de}$ & $210.7 \mathrm{~d}$ & $12.4 \mathrm{~d}$ & $15.09 \mathrm{de}$ & $30.1 \mathrm{~cd}$ \\
Calcáreo & Steiner & $206.3 \mathrm{~b}$ & $614.6 \mathrm{~b}$ & $886.7 \mathrm{~b}$ & $21 \mathrm{bc}$ & $69.21 \mathrm{~b}$ & $106.9 \mathrm{~b}$ \\
& Sólido & $89.03 \mathrm{~d}$ & $333.4 \mathrm{~cd}$ & $479.7 \mathrm{c}$ & $13.16 \mathrm{~cd}$ & $42.34 \mathrm{~cd}$ & $66.7 \mathrm{bcd}$ \\
& Vermicomposta & $32.2 \mathrm{e}$ & $65.8 \mathrm{e}$ & $81.6 \mathrm{~d}$ & $5.39 \mathrm{~d}$ & $8.8 \mathrm{e}$ & $11.4 \mathrm{~d}$ \\
\hline
\end{tabular}

Significancia de $p \leq 0.05$ DLS Fisher. 
Los tratamientos de solución nutritiva Steiner y de fertilizantes sólidos para el suelo de bosque obtuvieron los valores más altos $4546 \mathrm{~g} \mathrm{planta}^{-1}$ y $3938 \mathrm{~g} \mathrm{planta}^{-1}$, mientras que el tratamiento de té de vermicomposta presentó valores promedio entre los dos suelos de $1355 \mathrm{~g}^{\text {planta }^{-1}}$ siendo $^{2}$ estos los más bajos del experimento.

Hernández-Díaz et al. (2014) encontró diferencias significativas en los niveles referenciales de nutrientes en la solución del suelo en un cultivo de tomate bajo condiciones protegidas en dos épocas de producción. Estas diferencias se presentaron en la concentración de iones en la solución del suelo como la acumulación de biomasa, en la floración durante la primavera (marzo-junio) reportó valores para el $\mathrm{NO}_{3}{ }^{-}$de $371.3 \mathrm{mg} \mathrm{L}^{-1}, \mathrm{Ca}^{2+} 58.2 \mathrm{mg} \mathrm{L}^{-1}, \mathrm{~K}^{+} 123.24 \mathrm{mg} \mathrm{L}{ }^{-1}, \mathrm{Mg}^{2+} 16.08 \mathrm{mg}$ $\mathrm{L}^{-1}, \mathrm{pH} 7.23$ y CE $1300 \mu \mathrm{S} \mathrm{cm}^{-1}$ con una producción de materia seca total $206.53 \mathrm{~g} \mathrm{~m}^{-2}$ ).

El tratamiento con Steiner para los dos tipos de suelos obtuvo la mayor producción total de materia seca de $557.01 \mathrm{~g} \mathrm{~m}^{-2}$, el tratamiento sólido $303.48 \mathrm{~g} \mathrm{~m}^{-2}$ y el de vermicomposta $91.86 \mathrm{~g} \mathrm{~m}^{-2}$ con un rendimiento promedio para el tratamiento con Steiner de $4746.22 \mathrm{~g} \mathrm{planta}^{-1}$, seguido del tratamiento con fertilizante sólido $3994.1 \mathrm{~g}$ planta $^{-1} \mathrm{y}$ el tratamiento con vermicomposta de 1050 g planta ${ }^{-1}$ para el suelo de bosque, en cuanto al suelo calcáreo el rendimiento fue menor (Figura 1).

Conductividad eléctrica de la solución del suelo. Desde el punto de vista dinámico, la conductividad eléctrica (CE) fue diferente tanto para las dos clases de suelo como para los tipos de fertilización (Figura 2a y 2b). Durante la fase de crecimiento vegetativo (primeros 40 ddt) la mayor CE se presentó en los tratamientos con solución Steiner y fertilizante sólido, mientras que el tratamiento con vermicomposta presentó los valores más bajos. La solución Steiner y el fertilizante sólido no mostraron, salvo en unos cuantos muestreos, diferencias entre los valores de CE, mientras que el tratamiento de vermicomposta mostró de forma casi constante valores más bajos.
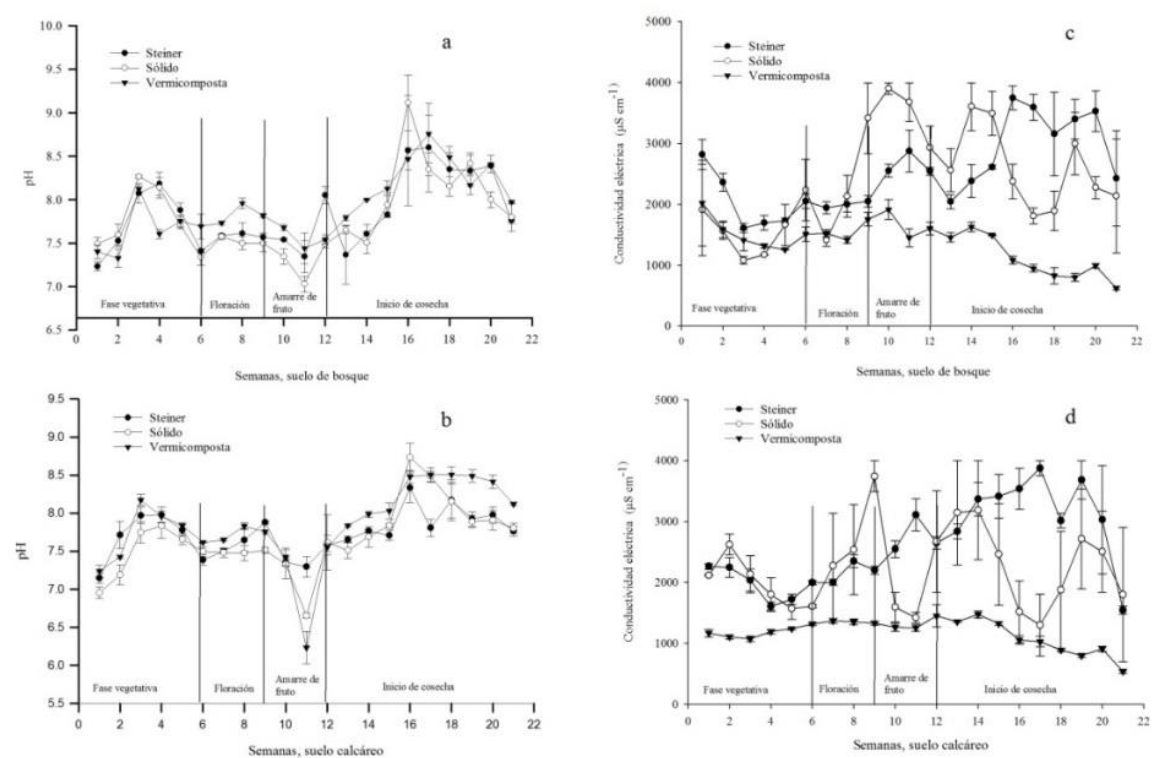

Figura 2. Valores medios y error estándar de la conductividad eléctrica y el pH en la solución del suelo a través del tiempo para dos clases de suelo con diferentes tipos de aplicación de fertilizantes en plantas de tomate. Las líneas verticales marcan etapas de desarrollo de las plantas. 
Durante la etapa reproductiva los valores de CE aumentaron para todos los tratamientos, esto independientemente al tipo de suelo, si bien el tratamiento de vermicomposta tuvo los valores más bajos (Figura 3a y 3b). Lo anterior, posiblemente porque el aporte de materia orgánica modifica el balance de intercambios iónicos en el suelo, teniendo como resultado un perfil diferente de iones disponibles, más que una diferente CE (Narváez-Ortiz et al., 2015).
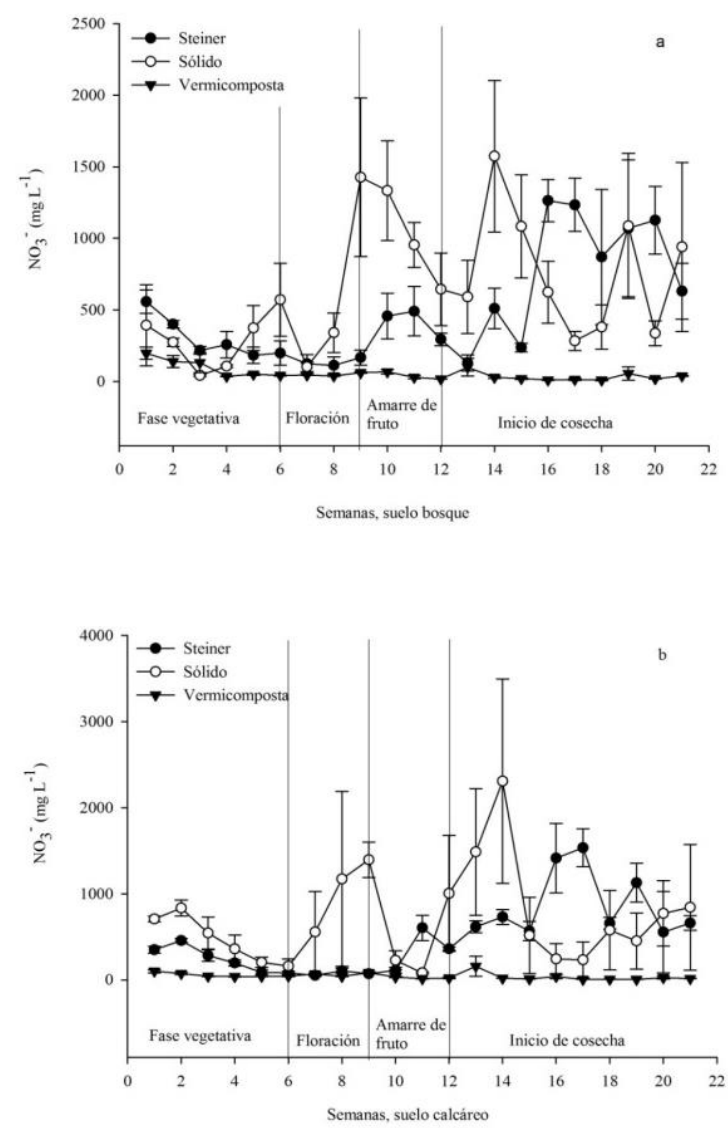

Figura 3. Valores medios y error estándar del $\mathrm{NO}_{3}{ }^{-}$en la solución del suelo a través del tiempo para dos clases de suelo con diferentes tipos de aplicación de fertilizantes en plantas de tomate. Las líneas verticales marcan etapas de desarrollo de las plantas.

Por otra parte, las diferencias en los valores de CE entre tratamientos dentro de cada tipo de suelo probablemente reflejan la distinta carga de nutrientes en la matriz de intercambio (Cuadro 2) y la resultante capacidad buffer de cada suelo, en interacción con las diferentes fuentes de fertilizantes (Chenu et al., 2000), adicionalmente, debe tomarse en cuenta el trabajo metabólico diferencial de las raíces (extrusión de protones o de ácidos orgánicos) en cada tipo de suelo, factor que también induce cambios en la composición del suelo (Broadley, 2012).

pH de la solución del suelo. En el suelo calcáreo el pH de la solución de suelo presentó un promedio de 7.8 (Figura 2c y 2d ). Este promedio fue inclusive menor al observado para el pH de la SS en los tres tratamientos aplicados en el suelo de bosque. De forma general, para los dos suelos y los diferentes tratamientos, el $\mathrm{pH}$ dismunuyó 7.6 durante la etapa vegetativa y durante la floración y amarre de fruto 7.5 y aumentó 8 de nuevo al llegar la cosecha de frutos, sin presentar diferencias significativas entre los tratamientos. 
El suelo de bosque presentó un pH menor que el suelo calcáreo (Cuadro 2), pero durante el experimento la solución del suelo del bosque mostró $\mathrm{pH}$ mayor al del suelo calcáreo (Figura 2c y 2d). Es posible que dicho efecto haya dependido de una mayor absorción de $\mathrm{NO}_{3}{ }^{-}$y otros iones por parte de la raíz (Butterly et al., 2013).

Los cambios observados en los valores del $\mathrm{pH}$ entre etapas de crecimiento de las plantas fueron debidas seguramente a cambios en la actividad metabólica de la planta, principalmente la absorción de $\mathrm{NO}_{3}{ }^{-}$, que originó modificaciones en los iones intercambiados con la solución del suelo (Broadley, 2012; Llanderal et al., 2019). Por otra parte, una explicación para la poca diferencia en los valores de $\mathrm{pH}$ entre tratamientos la señalan (Lao et al., 2003) quienes mencionan que la capacidad de amortiguamiento del suelo mantiene el $\mathrm{pH}$ dentro de ciertos límites inclusive utilizando soluciones nutritivas de diferente composición. Sin embargo, estos autores señalan que no ocurren diferencias entre etapas de crecimiento de las plantas de tomate, mientras que en el presente trabajo si ocurrieron, las diferencias fueron seguramente debidas al volumen de suelo utilizado, ya que Lao et al. (2003) realizaron su estudio en campo abierto, sin restricción en el volumen de suelo, mientras que en presente experimento se usaron macetas.

Nitrato en la solución del suelo. El valor promedio de concentración del $\mathrm{NO}_{3}{ }^{-}$en la $\mathrm{SS}$ de los tres tratamientos fue de $398.57 \mathrm{mg} \mathrm{L}^{-1}$, correspondiendo las concentraciones más altas a los tratamientos de fertilizante sólido y Steiner (promedio 500.92 y $640.52 \mathrm{mg} \mathrm{L}^{-1}$ respectivamente), mientras que el tratamiento con vermicomposta presentó un promedio de $54.25 \mathrm{mg} \mathrm{L}^{-1}$ (Figura 3a y 3b), cabe notar que el comportamiento dinámico del $\mathrm{NO}_{3}{ }^{-}$en la SS tanto en el suelo calcáreo como en el suelo de bosque, fue relativamente estable para el tratamiento de vermiccomposta.

El valor $48.17 \mathrm{mg} \mathrm{L}^{-1}$ obtenido para la vermicomposta (Figura 4a y 4b) es una concentración que se encuentra por debajo del umbral de $62 \mathrm{mg} \mathrm{L}^{-1}$ que se considera la mínima que induce transportadores de nitrato de baja afinidad (Dechorgnat et al., 2011). En los fenómenos de intercambio entre la solución y la matriz sólida del suelo el nitrato cumple un importante papel no solo como fuente de $\mathrm{N}$, sino en el equilibrio de cargas, por tratarse del anión más abundante en la SS; sin embargo, los valores muy altos pueden dar lugar a lixiviación por su gran movilidad, con la consiguiente contaminación del agua subterránea o bien a excesiva acumulación de nitrato en los tejidos vegetales (Dechorgnat et al., 2011; Hernández-Díaz et al., 2014; Llanderal et al., 2019).

Igualmente es notable una tendencia a mostrar valores más altos de concentración de $\mathrm{NO}_{3}{ }^{-}$en el período de cosecha, lo cual puede indicar alguna asociación entre los procesos físiológicos de la planta y la composición de la SS, posiblemente a través de los exudados radicales (Keiluweit et al., 2015). Por otra parte, una posible explicación basada en la tendencia a una mayor mineralización al variar el contenido relativo de agua del suelo (Plettp et al., 2020) no suena plausible, ya que no se permitió que el agua del suelo disminuyera en su disponibilidad para las plantas y se sabe que la mayor disponibilidad de nitrato ocurre en sistemas óxicos con poros del suelo con menor volumen de agua (Alvarez y Steinbach, 2009).

$\mathrm{N}$ total y $\mathrm{K}^{+}$en la solución del suelo. Los valores promedio para el $\mathrm{N}$ total en la SS fueron de 79, 81 y $79 \mathrm{mg} \mathrm{L}^{-1}$ para los tratamientos de solución Steiner, fertilizante sólido y vermicomposta, respectivamente. Al contrario que lo observado con el $\mathrm{NO}_{3}{ }^{-}$, el $\mathrm{N}$ total tuvo un proceso dinámico mucho más estable y sin diferencias significativas entre los tratamientos en ambos suelos (Figura 4a y 4b). Cabe resaltar, que en ambos tipos de suelo los valores más bajos de $\mathrm{N}$ total se presentaron en la fase de la cosecha de fruto; es decir, al contrario que lo observado para el $\mathrm{NO}_{3}{ }^{-}$(Figura 3). 

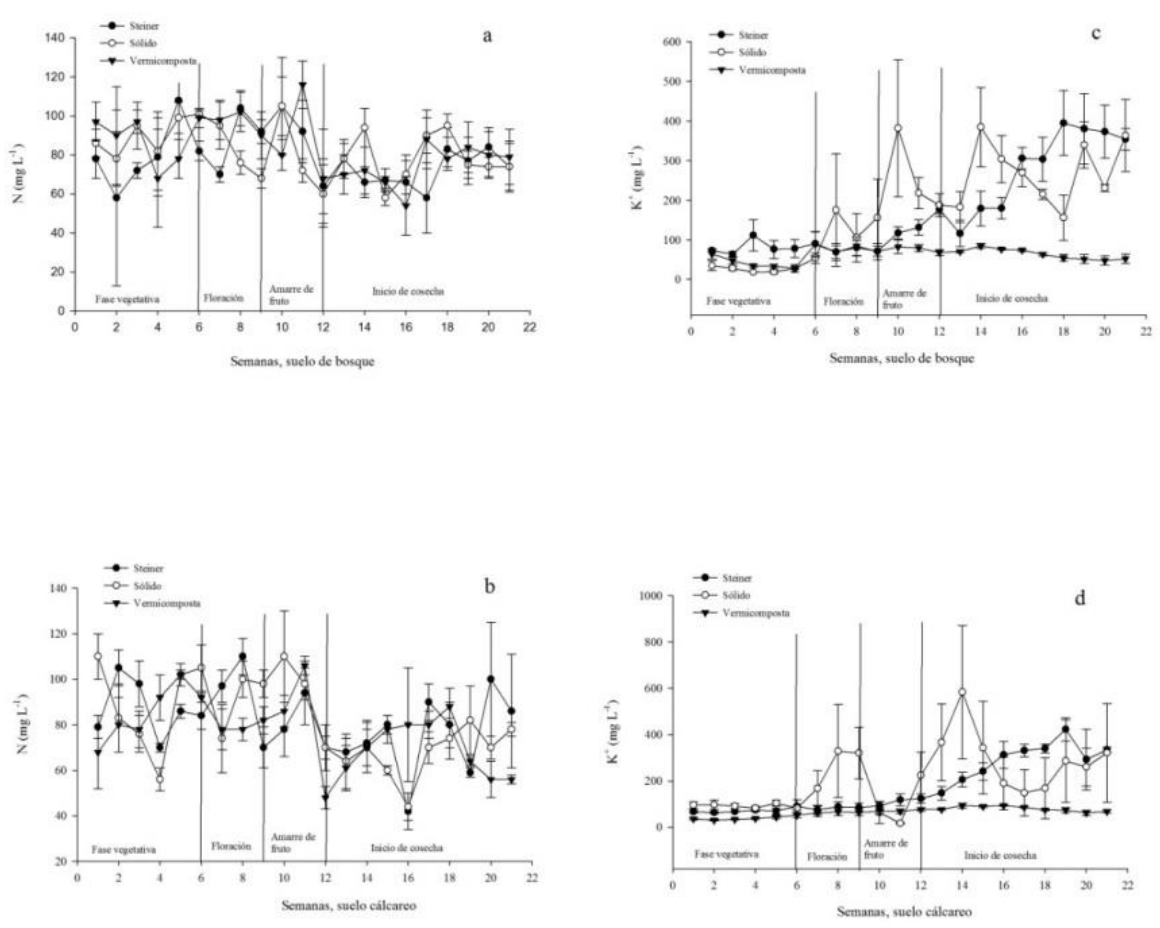

Figura 4. Valores medios y error estándar de la concentración de $\mathbf{N}$ total y $\mathrm{K}^{+}$en la solución del suelo a través del tiempo para dos clases de suelo con diferentes tipos de aplicación de fertilizantes en plantas de tomate. Las líneas verticales marcan etapas de desarrollo de las plantas.

En cuanto a la concentración del $\mathrm{K}^{+}$el promedio para el suelo de bosque y el suelo calcáreo fue de 256 y $241 \mathrm{mg} \mathrm{L}^{-1}$, respectivamente. El comportamiento dinámico del $\mathrm{K}^{+}$en la SS fue diferente para cada tratamiento (Figura 4c y 4d).

El tratamiento de vermicomposta mostró valores estables, pero con una pequeña tendencia a elevarse en las fases de floración y llenado del fruto, mostrando un promedio general de concentración de $\mathrm{K}^{+}$de $60 \mathrm{mg} \mathrm{L}^{-1}$. En cambio, el tratamiento con solución Steiner presentó una tendencia positiva constante, alcanzando los valores más altos durante la cosecha de frutos y presentando un promedio general de $372 \mathrm{mg} \mathrm{L}^{-1}$ de $\mathrm{K}^{+}$. El tratamiento de fertilizantes sólidos alcanzó los valores más altos durante la etapa de cosecha para los dos tipos de suelo y un promedio general de $361 \mathrm{mg} \mathrm{L}^{-1}$.

La variación en las cantidades de N (Figura 5a y 5b) en las diferentes etapas del ciclo del cultivo pudiera indicar alguna asociación entre los procesos químicos y biológicos que aportan diferentes formas de $\mathrm{N}$ a la SS y el comportamiento fisiológico de la planta, en este caso posiblemente la tasa de exudación de metabolitos por la raíz (Keiluweit et al., 2015). o por el aumento en la tasa de mineralización que ocurre frente al mayor contenido relativo de agua (Plett et al., 2020).

El tratamiento con fertilizante sólidos presentó una tendencia positiva al igual que el tratamiento con Steiner en la dinámica del $\mathrm{K}^{+}$(Figura 5 c y 5d) en la SS, pero con picos de concentración que se presentaron después de la floración. Pudiera esperarse que la tendencia positiva en la 
disponibilidad de potasio de un mayor contenido relativo de agua del suelo, pero la misma tendencia debería de observarse en el tratamiento en donde se aplicó vermicomposta, sin que ocurriera así. Es probable entonces que el aporte adicional de materia orgánica de este último fertilizante modificara la adsorción del $\mathrm{K}^{+}$en los coloides del suelo (Yanai el al., 1997; Chenu et al., 2000; Yin el al., 2019).
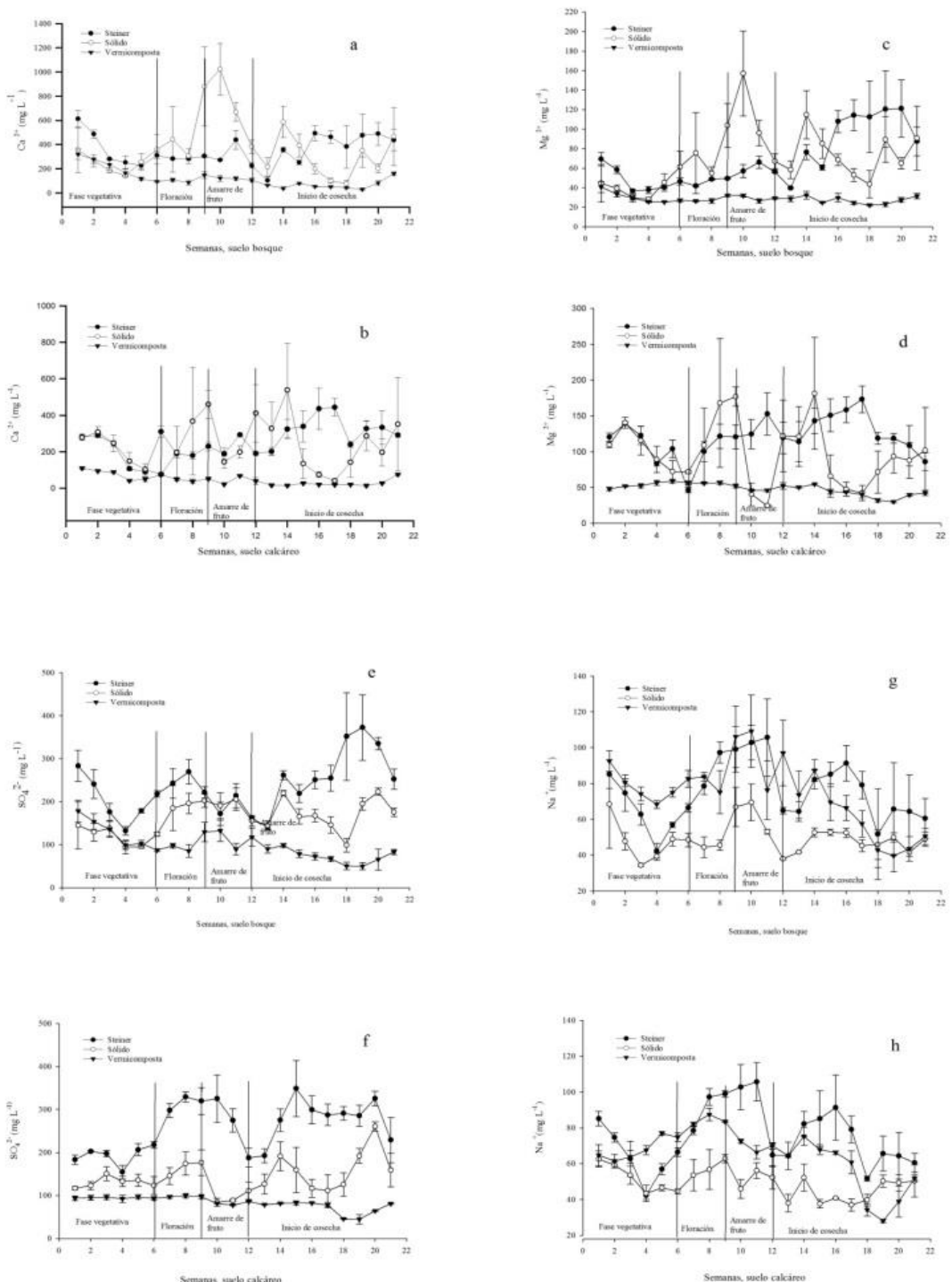

Figura 5. Valores medios y error estándar de la concentración de $\mathrm{Ca}^{2+}, \mathrm{Mg}^{2+}, \mathrm{SO}_{4}^{-2} \mathrm{y} \mathrm{Na}^{+}$en la solución del suelo a través del tiempo para dos clases de suelo con diferentes tipos de aplicación de fertilizantes en plantas de tomate. Las líneas verticales marcan etapas de desarrollo de las plantas.

$\mathrm{Ca}^{2+}, \mathrm{Mg}^{2+}, \mathrm{Na}^{+}$y $\mathrm{SO}_{4}{ }^{-2}$ en la solución del suelo. Los valores promedio de concentración del $\mathrm{Ca}^{2+}$, $\mathrm{Mg}^{2+}, \mathrm{Na}^{+}$y $\mathrm{SO}_{4}{ }^{-2}$ en la SS para cada tipo de suelo y tratamiento se muestran en la Cuadro 4. Cabe remarcar, que el comportamiento dinámico del $\mathrm{Ca}^{2+}$ y el $\mathrm{Mg}^{2+}$ (Figura 5) fue parecido al mostrado por el $\mathrm{K}^{+}$(Figura 4). Los valores más altos para la concentración de calcio se encontraron con la aplicación de fertilizante sólido. 
Los valores más altos para la concentración de calcio se encontraron con la aplicación de fertilizante sólido (Figura 5a y 5b), al comparar las fluctuaciones del $\mathrm{Ca}^{2+}$ con las del $\mathrm{Mg}^{2+}$ es posible observar una mayor estabilidad alrededor del promedio por parte del $\mathrm{Mg}^{2+}$ por lo que parece tratarse de un comportamiento asociado a los procesos de intercambio de estos cationes mayores, mostrando el $\mathrm{Ca}^{2+}$ una mayor cantidad de picos con mayor concentración y un rango de variación de cientos de $\mathrm{mg} \mathrm{L}^{-1}$ en comparación con el $\mathrm{Mg}^{2+}$ (Figura 5 c y $5 \mathrm{~d}$ ) que mostró un rango de variación de decenas de $\mathrm{mg} \mathrm{L}^{-1}$, con las variaciones más amplias de nuevo para el fertilizante sólido.

El comportamiento dinámico del $\mathrm{Na}^{+}$(Figura $5 \mathrm{~g}$ y $5 \mathrm{~h}$ ) fue diferente al del $\mathrm{Ca}^{2+}$ y con cierto parecido al del $\mathrm{Mg}^{2+}$, mostrando fluctuaciones y picos de concentración de decenas de $\mathrm{mg} \mathrm{L}^{-1}$, pero mostrando ahora los valores más bajos en el fertilizante sólido y los mayores para la solucion Steiner y la vermicomposta. En este último caso el resultado se explica por la gran cantidad de sodio encontrado de origen en el fertilizante (Yanai el al., 1996; Venterink el al., 2002; Sana et al., 2013).

En cuanto a los valores del $\mathrm{SO}_{4}{ }^{-2}$ (Figura 5 e y 5 f), para ambos suelos los más altos se encontraron durante la cosecha para el tratamiento Steiner. Una explicación parcial de este hecho podría ser el aporte de $\mathrm{H}_{2} \mathrm{SO}_{4}$ utilizado para la acidificación de la solución nutritiva.

Acumulación de biomasa vegetal. Los valores promedios de la biomasa fresca y seca acumulada para cada tipo de suelo y tratamiento a los 40,60 y 90 después del trasplante se describen en la Cuadro 4. La acumulación de biomasa mostró diferencias significativas $(p \leq 0.05)$, con los valores más altos en los tratamientos de Steiner y fertilizante sólido mientras que los valores más bajos en el tratamiento con vermicomposta.

La composición de la solución del suelo se vio afectada por el crecimiento de la planta es decir la composición de los aniones y cationes, así como sus concentraciones mostraron cambios dinámicos dependientes del crecimiento de la planta (Figuras 1-5). Este mismo fenómeno fue reportado por (Hernández-Díaz et al., 2014) en un cultivo detomate.

Con un suministro adecuado de nutrientes, los cultivos de crecimiento rápido tienen mayor tasa de captación de nutrientes por unidad de peso de raíz, lo que origina diferencias en la absorción e intercambio de iones, modificando a su vez la composición de la solución del suelo de una manera diferenciada, dependiendo de la biomasa radical y de la actividad metabólica de la misma, esta conclusión fue corroborada por (Christie y Moorby, 1975) que investigaron diferentes pastos bajo condiciones controladas en invernadero. Es difícil distinguir si en los cultivos de rápido crecimiento ocurre mayor absorción por unidad de biomasa radical (tal vez por mayor densidad de canales por unidad de superficie radical) o bien si esta respuesta depende de una mayor biomasa radical por planta ( Hamnér et al., 2017).

\section{Conclusiones}

La dinámica de los iones en la solución del suelo a través del tiempo y la acumulación de biomasa así como su composición química se ve afectada por la concentración de iones en los dos tipos de suelo con las tres formas de aplicación de los fertilizantes, en donde los valores más altos se presentaron con el tratamiento con la solución Steiner. 


\section{Literatura citada}

Alvarez, R. and Steinbach, H. S. 2009. A review of the effects of tillage systems on some soil physical properties, water content, nitrate availability and crops yield in the argentine pampas. Netherlands. Soil and Tillage Res. 104(1):1-15. Doi: 10.1016/j.still.2009.02.005.

Broadley, M.; Brown, P.; Cakmak, I.; Rengel, Z. and Zhao, F. 2012. Function of nutrients: micronutrients. In Marschner's mineral nutrition of higher plants . Academic Press. 191248 pp. Doi:10.1016/B978-0-12-384905-2.00007-8.

Butterly, C. R.; Baldock, J. A. and Tang, C. 2013. The contribution of crop residues to changes in soil $\mathrm{pH}$ under field conditions. Netherlands. Plant and Soil. 366(1):185-198. doi:10.1007/s11104-012-14221.

Chenu, C.; Bissonnais, Y. L. and Arrouay, D. 2000. Organic matter influence on clay wettability and soil aggregate stability. United States. Soil Sci. Soc. Am. J. 64(4):1479-1486. Doi: 10.2136/sssaj2000.6441479x.

Christie, E. K. and Moorby, J. 1975. Physiological responses of semiarid grasses. I. The influence of phosphorus supply on growth and phosphorus absorption. Australia. Australian J. Agric. Res. 26(3):423-436. Doi: 10.1071/AR9750423.

Dechorgnat, J.; Nguyen, C. T.; Armengaud, P.; Jossier, M.; Diatloff, E.; Filleur, S. and Daniel, F. 2011. From the soil to the seeds: the long journey of nitrate in plants. Great Britain. J. Exp. Bot. 62(4):1349-1359. Doi.org/10.1093/jxb/erq409.

Hamnér, K.; Weih, M.; Eriksson, J. and Kirchmann, H. 2017. Influence of nitrogen supply on macro-and micronutrient accumulation during growth of winter wheat. Netherlands. Field Crops Res. 213(2017):118-129. Doi:10.1016/j.fcr.2017.08.002.

Hermans, C.; Hammond, J. P.; White, P. J. and Verbruggen, N. 2006. How do plants respond to nutrient shortage by biomass allocation. England. Trends in Plant Science. 11(12):610-617. Doi: $10.1016 /$ j.tplants.2006.10.007.

Hernández-Díaz, M. I.; Laffita, M. C.; Moreno-Placeres, V.; Igarza-Sánchez, A. y Ojeda-Veloz, A. 2014. Niveles referenciales de nutrientes en la solución del suelo para el diagnóstico nutricional en el cultivo protegido del tomate. Chile. Idesia (Arica). 32(2):79-88. Doi: 10.4067/S0718-34292014000200011.

Huang, P. M.; Li, Y. and Sumner, M. E. 2011. Handbook of soil sciences: properties and processes. $2^{\mathrm{a}}$ (Ed). CRC Press Taylor \& Francis Group. Boca Raton. Florida. USA. 351-371 pp.

Keiluweit, M.; Bougoure, J. J.; Nico, P. S.; Pett-Ridge, J.; Weber, P. K. and Kleber, M. 2015. Mineral protection of soil carbon counteracted by root exuddtes. England. Nature Climate Change. 5(6):588-595. Doi: 10.1038/nclimate2580.

Lao, M. T.; Jiménez, S.; Eymar, E.; Fernández, E. J. and Jiménez, R. 2003. Determination of spatial variability of nutrient composition of soil solutions in greenhouses by using suction cups. United States. Communications in Soil Science and Plant Analysis. 34(5-6):865-879. Doi: 10.1081/CSS-120018980.

Llanderal, A.; García-Caparrós, P.; Contreras, J. I.; Segura, M. L. and Teresa Lao, M. 2019. Spatiotemporal variations in nutrient concentration in soil solution under greenhouse tomato. United States. J. Plant Nutr. 42(8):842-852. doi: 10.1080/ 01904167.2019.1584219.

Narváez-Ortiz, W. A.; Morales-Díaz, A. B.; Benavides-Mendoza, A. y Reyes-Valdés M. H. 2015. Dinámica de la composición de la solución del suelo en cultivos del occidente de méxico. México. Rev. Mex. Cienc. Agríc. 12(6):2383-2397. 
Plett, D. C.; Ranathunge, K.; Melino, V. J.; Kuya, N.; Uga, Y. and Kronzucker, H. J. 2020. The intersection of nitrogen nutrition and water use in plants: new paths toward improved crop productivity. Great Britain. J. Exp. Bot. 71(15):4452-4468. Doi:10.1093/jxb/eraa049.

Sana, A.; Sarah, S.; Asim, M.; Almas, H. and Saamia, S. 2013. Comparative analysis of animal manure for soil conditioning. Australia. Inter. J. Agron. Plant Produc. 4(12):3360-3366.

SEMARNAT. 2000. Norma Oficial Mexicana NOM-021. Que establece las especificaciones de fertilidad, salinidad y clasificación de suelos. Registro que acompaña a las muestras desde su obtención hasta su entrega al laboratorio de pruebas y análisis.

Souza, E. R. D.; Melo, H. F. D.; Almeida, B. G. D. y de Melo, D. V. 2013. Comparação de métodos de extração da solução do solo. Brasil. Revista Brasileira de Engenharia Agrícola e Ambiental. 17(5):510-517. Doi:10.1590/S1415-43662013000500007.

Venterink, H. O.; Davidsson, T. E.; Kiehl, K. and Leonardson, L. 2002. Impact of drying and rewetting on n, p and k dynamics in a wetland soil. Netherlands. Plant and Soil. 243(1):119130. doi: 10.1023/A:1019993510737.

Yanai, J.; Linehan, D. J.; Robinson, D.; Young, I. M.; Hackett, C. A.; Kyuma, K. and Kosaki, T. 1996. Effects of Inorganic nitrogen application on the dynamics of the soil solution composition in the root zone of maize. Netherlands. Plant and Soil. 180(1):1-9. doi: 10.1007/BF00015405.

Yanai, J.; Kosaki, T.; Nakano, A. and Kyuma, K. 1997. Application effects of controlledavailability fertilizer on dynamics of soil solution composition. United States. Science Society of America Journal. 61(6):1781-1786. doi: 10.2136/sssaj1997.03615995006 $100060033 x$.

Yin, Q.; Tian, T.; Han, X.; Xu, J.; Chai, Y.; Mo, J. and Yue, M. 2019. The relationships between biomass allocation and plant functional trait. United States. Ecological Indicators. 102. 302308. doi.org/10.1016/j.ecolind.2019.02.047. 